\title{
Asymptotic Distribution of Critical Transmission Radius for Greedy Forward Routing
}

\author{
Peng-Jun Wan \\ Department of Computer Science \\ Illinois Institute of Technology \\ Chicago, IL 60616 \\ Email:wan@cs.iit.edu
}

\author{
Lixin Wang \\ Department of Math and Computer Science \\ Paine College \\ Augusta, GA 30901 \\ Email: lwang@paine.edu
}

\begin{abstract}
Consider a random multihop wireless network represented by a Poisson point process over a unit-area disk with mean $n$. Let $\phi_{n}$ denote its critical transmission radius for its greedy forward routing. Recently, asymptotic bounds on $\phi_{n}$ have been progressively improved. However, the precise asymptotic probability distribution of $\phi_{n}$ remains open. In this paper, we settle this open problem. Specifically, let $\sigma=\frac{2 \pi}{3}-\frac{\sqrt{3}}{2}$. Then for any constant $c$, the asymptotic probability of $\phi_{n} \leq \sqrt{\frac{\ln n+c}{\sigma n}}$ is proved to be exactly $\exp \left(-\left(\frac{1}{\sigma / \pi-1 / 3}-\frac{\pi}{2 \sigma}\right) e^{-c}\right)$.

Index Terms-Greedy forward routing, critical transmission radius, random deployment, asymptotic distribution.
\end{abstract}

\section{INTRODUCTION}

Greedy forward routing (GFR) in multihop wireless networks is a classical geographic routing in which each node discards a packet if none of its neighbors is closer to the destination of the packet than itself, or otherwise forwards the packet to the neighbor closest to the destination of the packet. GFR only requires each node to maintain the locations of its one-hop neighbors and each packet to contain the location of its destination node. Thus, it can be implemented in a localized and memoryless manner. However, GFR may discard a packet before it reaches its destination if the transmission radii of the nodes are small. To ensure that every packet can reach its destination, all nodes should have sufficiently large transmission radii. The critical transmission radius (CTR) of a planar node set $V$ for GFR is the smallest transmission radius by $V$ which ensures successful delivery of any packets from any source node in $V$ to any destination node in $V$. It is explicitly given by

$$
\max _{u \in V} \max _{v \in V} \min \{\|w u\|:\|w v\| \leq\|u v\|, w \in V \backslash\{u\}\},
$$

where $\|u v\|$ denotes the Euclidean distance between two nodes $u$ and $v$.
Denote by $\mathcal{P}_{n}$ the Poisson point process over a unit-area disk with density $n$, and by $\phi_{n}$ the CTR of $\mathcal{P}_{n}$ for GFR. The analytic study of GFR can date back to 1978 by Kleinrock and Silvester [5]. Little is known about the asymptotics of $\phi_{n}$ until the study in 2006 by Wan et al. [11]. Denote by $\sigma$ the geometric constant $\frac{2 \pi}{3}-\frac{\sqrt{3}}{2}$ (whose geometric meaning will be explained in the later section). It was proved in [11] that for any constant $\varepsilon>0$, it is asymptotically almost sure (abbreviated by a.a.s.) that

$$
(1-\varepsilon) \sqrt{\frac{\ln n}{\sigma n}} \leq \phi_{n} \leq(1+\varepsilon) \sqrt{\frac{\ln n}{\sigma n}} .
$$

However, the asymptotic probability distribution of $\phi_{n}$ was still unresolved. A recent progress was made by Wang et al. [14]. Fix a constant $c$ and let

$$
r_{n}=\sqrt{\frac{\ln n+c}{\sigma n}} .
$$

It was shown in [14] that the asymptotic probability of $\phi_{n} \leq r_{n}$ is at least $1-\left(\frac{1}{\sigma / \pi-1 / 3}-\frac{\pi}{2 \sigma}\right) e^{-c}$ and at most $e^{-\frac{\pi}{2 \sigma} e^{-c}}$. As an immediate consequence, for any positive sequence $\left(c_{n}: n \geq 1\right)$ with $c_{n}=o(\ln n)$ and $c_{n} \rightarrow \infty$, it is asymptotically almost sure that

$$
\sqrt{\frac{\ln n-c_{n}}{\sigma n}}<\phi_{n} \leq \sqrt{\frac{\ln n+c_{n}}{\sigma n}} .
$$

We remark that the above inequalities are stronger than those given in equation (1). But the precise asymptotic probability distribution of $\phi_{n}$ remains open.

In this paper, we derive the precise asymptotic probability distribution of $\phi_{n}$ and prove that the conjecture stated in [14] is true. Let

$$
\mu=\left(\frac{1}{\sigma / \pi-1 / 3}-\frac{\pi}{2 \sigma}\right) e^{-c} .
$$

The main result of this paper is stated in the following theorem. 
Theorem 1: $\lim _{n \rightarrow \infty} \operatorname{Pr}\left[\phi_{n} \leq r_{n}\right]=e^{-\mu}$.

Our proof for the above theorem only uses two basic tools from the probability theory: the Palm theory for Poisson processes and the Brun's sieve. The innovative part of our proof is the subtle partition of the event $\phi_{n} \leq r_{n}$ into a number of special events whose asymptotic probabilities can be computed easily. We believe our approach is quite general and can be applied to obtain the critical transmission radii of other variants of greedy forward routing proposed in [1], [2], [3], [4], [6], [7], [9], [15].

In what follows, $\mathbf{o}$ is origin of the Euclidean plane $\mathbb{R}^{2}$, and $\mathbb{D}$ is the unit-area (closed) disk centered at o. We assume that $\mathcal{P}_{n}$ is the Poisson point process over $\mathbb{D}$ with density $n$. We denote by $\mathcal{X}_{n}=\left(X_{1}, \cdots, X_{n}\right)$ the uniform $n$-point process over $\mathbb{D}$. The symbols $O, o, \sim$ always refer to the limit $n \rightarrow \infty$. To avoid trivialities, we tacitly assume $n$ to be sufficiently large if necessary. For simplicity of notation, the dependence of sets and random variables on $n$ will be frequently suppressed. For any finite set $S, \operatorname{card}(S)$ denotes the cardinality of $S$. For any set $S$ and positive integer $k$, the $k$-fold Cartesian product of $S$ is denoted by $S^{k}$. The Euclidean norm of a point $x$ is denoted by $\|x\|$, and the Euclidean distance between two points $u$ and $v$ is denoted by $\|u v\|$. The Lebesgue measure (or area) of a measurable set $A \subset \mathbb{R}^{2}$ is denoted by $|A|$. The topological boundary of a set $A \subset \mathbb{R}^{2}$ is denoted by $\partial A$. The open (respectively, closed) disk of radius $r$ centered at $x$ is denoted by $D(x, r)$ (respectively, $\bar{D}(x, r)$ ).

The remaining of this paper is organized as follows. The proof for Theorem 1 is presented in Section III. Some preliminary results to be used in the proof for Theorem 1 are established in Section II. The proof for a technical lemma in Section II on the limits of two relevant integrals is postponed in Section IV.

\section{PRELIMINARIES}

For any pair of points $u$ and $v$ on the plane, define

$$
L(u, v)=D(u,\|u v\|) \cap D(v,\|u v\|) .
$$

The set $L(u, v)$ is called a lune of $u$ and $v$ (see Figure 1(a)). It's easy to verify that

$$
|L(u, v)|=\sigma\|u v\|^{2} .
$$

Thus, $\sigma$ is the area of the lune of two points with unit distance.

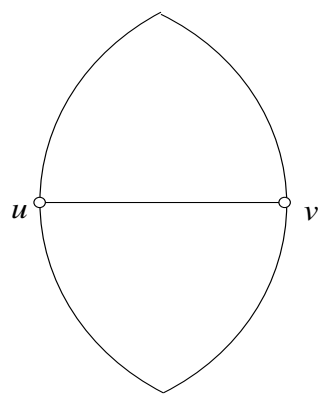

(a)

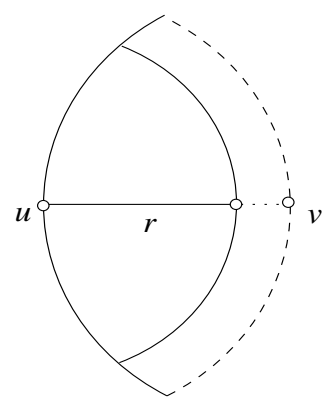

(b)
Fig. 1. Illustrations for (a) $L(u, v)$ and (b) $L_{r}(u, v)$.

Let $r$ be a positive number. For any two points $u$ and $v$, define

$$
\begin{aligned}
& L_{r}(u, v)=D(u, r) \cap D(v,\|u v\|), \\
& \nu_{r}(u, v)=\min \left\{\left|L_{r}(u, v) \cap \mathbb{D}\right|,\left|L_{r}(v, u) \cap \mathbb{D}\right|\right\} .
\end{aligned}
$$

Figure 1(b) illustrates the region of $L_{r}(u, v)$ with $r \leq\|u v\|$. It's easy to verify that if $\|u v\| \leq r / 2$, then

$$
\left|L_{r}(u, v)\right|=\pi\|u v\|^{2}
$$

and otherwise,

$$
\begin{aligned}
\left|L_{r}(u, v)\right|= & 2\|u v\|^{2} \arcsin \frac{r}{2\|u v\|}+r^{2} \arccos \frac{r}{2\|u v\|} \\
& -r^{2} \sqrt{\left(\frac{\|u v\|}{r}\right)^{2}-\frac{1}{4}} .
\end{aligned}
$$

A geometric graph is a graph on a finite planar set whose edges are line segments. Let $V$ be a finite planar set. We use $K(V)$ to denote the complete geometric graph over $V$. Suppose that $r$ is a positive number. We use $G F R_{r}(V)$ to denote the geometric graph on $V$ in which there is an edge between two nodes $u$ and $v$ if and only if $\|u v\|>r$ and either $L_{r}(u, v) \cap V=\emptyset$ or $L_{r}(v, u) \cap V=\emptyset$. The $r$-disk graph of $V$ is a geometric graph over $V$ which consists of all edges $u v$ satisfying that $\|u v\| \leq r$.

Let $H$ be a nonempty geometric graph. We use $V(H)$ and $E(H)$ to denote the vertex set and edge set of $H$ respectively. An orientation of a graph $H$ is a digraph obtained from $H$ by orienting each edge of $H$ into an arc. Clearly, if $H$ has $k$ edges, then $H$ has $2^{k}$ orientations. Suppose that $r$ is a positive number. Let $\chi_{r}(H)$ be the indicator for $H \subseteq G F R_{r}(V(H))$. If $\chi_{r}(H)=1$, we use $\mathcal{D}(H)$ to denote the set of orientations $H^{\prime}$ of $H$ satisfying that $L_{r}(u, v) \cap V(H)=\emptyset$ for each arc $(u, v)$ of $H^{\prime}$, and define

$$
\nu_{r}(H)=\min _{H^{\prime} \in \mathcal{D}(H)}\left|\left(\cup_{(u, v) \in E\left(H^{\prime}\right)} L_{r}(u, v)\right) \cap \mathbb{D}\right| .
$$


Suppose that $n$ is a positive integer. For the pair of points $u$ and $v$, define

$$
\begin{aligned}
g_{n}(u, v) & =e^{-n\left|L_{r_{n}}(u, v) \cap \mathbb{D}\right|}+e^{-n\left|L_{r_{n}}(v, u) \cap \mathbb{D}\right|} \\
& -e^{-n\left|\left(L_{r_{n}}(u, v) \cup L_{r_{n}}(v, u)\right) \cap \mathbb{D}\right|} .
\end{aligned}
$$

Intuitively, $g_{n}(u, v)$ is the probability of the event that either $L_{r_{n}}(u, v) \cap \mathcal{P}_{n}=\emptyset$ or $L_{r_{n}}(v, u) \cap \mathcal{P}_{n}=\emptyset$ when $\|u v\| \geq r_{n}$. For any nonempty geometric graph $H$, define

$$
\begin{aligned}
& f_{n}(H)= \begin{cases}0, & \text { if } \chi_{r_{n}}(H)=0 ; \\
e^{-n \nu_{r_{n}}(H)}, & \text { if } \chi_{r_{n}}(H)=1 ;\end{cases} \\
& g_{n}(H)=\prod_{(u, v) \in E(H)} g_{n}(u, v) .
\end{aligned}
$$

Let $\varepsilon=0.001$. Fix a sequence $\left(c_{n}\right)$ of real numbers satisfying that $c_{n}>c, c_{n}=o(\ln \ln n)$ and $c_{n} \rightarrow \infty$. Let

$$
\begin{aligned}
& R_{n}=\sqrt{\frac{\ln n+c_{n}}{\sigma n}}, \\
& R_{n}^{\prime}=\left(1+\frac{\varepsilon}{2}\right) \sqrt{\frac{\ln n}{\sigma n}} .
\end{aligned}
$$

Then, for sufficiently large $n$,we have $r_{n}<R_{n}<R_{n}^{\prime}<$ $(1+\varepsilon) r_{n}$. Define

$$
\begin{aligned}
\Omega & =\left\{\left(x_{1}, x_{2}\right) \in \mathbb{D}^{2}: r_{n}<\left\|x_{1} x_{2}\right\| \leq R_{n}\right\}, \\
\Omega^{\prime} & =\left\{\left(x_{1}, x_{2}\right) \in \mathbb{D}^{2}: R_{n}<\left\|x_{1} x_{2}\right\| \leq R_{n}^{\prime}\right\} .
\end{aligned}
$$

The following lemma was proved in [14] (see Section 3 in [14]).

Lemma 2: The following asymptotic equalities are true:

$$
\begin{aligned}
n^{2} \int_{\Omega} e^{-n \nu_{r_{n}}\left(x_{1}, x_{2}\right)} d x_{1} d x_{2} & \sim \frac{e^{-c}}{\sigma / \pi-1 / 3}, \\
n^{2} \int_{\Omega} g_{n}\left(x_{1}, x_{2}\right) d x_{1} d x_{2} & \sim 2 \mu, \\
n^{2} \int_{\Omega^{\prime}} g_{n}\left(x_{1}, x_{2}\right) d x_{1} d x_{2} & =o(1) .
\end{aligned}
$$

A topology with numbered vertices is specified by a collection of the pairs of the indices of the numbered vertices. For any integer $m \geq 2$, denote $\mathcal{T}_{m}$ set of topologies on $m$ numbered vertices without isolated vertex. For any $\tau \in \mathcal{T}_{m}$, and any sequence $U$ of $m$ planar points, $\tau(U)$ denotes the geometric graph on $U$ with topology $\tau$. For any $\tau \in \mathcal{T}_{m}$, we denote by $\Gamma(\tau)$ the set of $\mathbf{x} \in \mathbb{D}^{m}$ satisfying that all edges of $\tau(\mathbf{x})$ have length in $\left(r_{n}, R_{n}\right]$. Note that for each $\mathbf{x} \in \Gamma(\tau)$, the $\sqrt{3} R_{n}$-disk graph on the midpoints of the edges in any connected component of $\tau(\mathbf{x})$ is connected. Thus, the $\sqrt{3} R_{n^{-}}$ disk graph on the midpoints of the edges in $\tau(\mathbf{x})$ has no more connected components than $\tau(\mathbf{x})$ itself. For any positive integer $l$ not larger than the number of connected components of $\tau$, we denote by $\Gamma_{l}(\tau)$ the set of $\mathbf{x} \in \Gamma(\tau)$ such that the $\sqrt{3} R_{n}$-disk graph on the midpoints of the edges in $\tau(\mathbf{x})$ has $l$ connected components.

Lemma 3: Suppose that $2<m \leq 2 k$ and $\tau$ is a forest in $\mathcal{T}_{m}$ with $k$ edges. Then, for any positive integer $l \leq$ $\min \{m-k, k-1\}$,

$$
\begin{aligned}
& n^{m} \int_{\Gamma_{l}(\tau)} f_{n}(\tau(\mathbf{x})) d \mathbf{x}=o(1), \\
& n^{m} \int_{\Gamma_{l}(\tau)} g_{n}(\tau(\mathbf{x})) d \mathbf{x}=o(1) .
\end{aligned}
$$

The proof for Lemma 3 is postponed to Section IV. Lemma 3 implies the following two corollaries.

Corollary 4: Suppose that $\tau \in \mathcal{T}_{2 k}$ is a perfect matching for some $k \geq 2$. Then,

$$
n^{2 k} \int_{\Gamma_{k}(\tau)} g_{n}(\tau(\mathbf{x})) d \mathbf{x} \sim(2 \mu)^{k}
$$

Proof: We denote by $C_{k}$ the perfect matching of $2 k$ numbered vertices $v_{1}, v_{2}, \cdots, v_{2 k}$ which consists of $k$ edges $v_{2 i-1} v_{2 i}$ for $1 \leq i \leq k$. By symmetry, we only have to prove the lemma holds for $\tau=C_{k}$. Note that

$$
\Gamma_{k}\left(C_{k}\right)=\Gamma\left(C_{k}\right) \backslash \cup_{l=1}^{k-1} \Gamma_{l}\left(C_{k}\right) .
$$

Since $\Gamma\left(C_{k}\right)=\Omega^{k}$, we have

$$
\begin{aligned}
& n^{2 k} \int_{\Gamma\left(C_{k}\right)} g_{n}\left(C_{k}(\mathbf{x})\right) d \mathbf{x} \\
= & \prod_{i=1}^{k}\left(n^{2} \int_{\Omega} g_{n}\left(x_{2 i-1}, x_{2 i}\right) d x_{2 i-1} d x_{2 i}\right) \\
\sim & (2 \mu)^{k},
\end{aligned}
$$

By Lemma 3 for each $1 \leq l \leq k-1$,

$$
n^{2 k} \int_{\Gamma_{l}\left(C_{k}\right)} g_{n}\left(C_{k}(\mathbf{x})\right) d \mathbf{x}=o(1) .
$$

Thus, the corollary holds.

Corollary 5: Suppose that $\tau \in \mathcal{T}_{m}$ is not a perfect matching. Then,

$$
n^{m} \int_{\Gamma(\tau)} f_{n}(\tau(\mathbf{x})) d \mathbf{x}=o(1) .
$$


Proof: Clearly, $m>2$. Let $\tau^{\prime}$ be a maximal spanning forest of $\tau$. Then, $\tau^{\prime} \in \mathcal{T}_{m}$ and $\tau^{\prime}$ is not a perfect matching. In addition, $\Gamma\left(\tau^{\prime}\right) \supseteq \Gamma(\tau)$, and for any $\mathbf{x} \in \Gamma(\tau)$,

$$
f_{n}\left(\tau^{\prime}(\mathbf{x})\right) \geq f_{n}(\tau(\mathbf{x})) .
$$

Hence,

$$
\int_{\Gamma(\tau)} f_{n}(\tau(\mathbf{x})) d \mathbf{x} \leq \int_{\Gamma(\tau)} f_{n}\left(\tau^{\prime}(\mathbf{x})\right) d \mathbf{x} \leq \int_{\Gamma\left(\tau^{\prime}\right)} f_{n}\left(\tau^{\prime}(\mathbf{x})\right) d \mathbf{x} .
$$

Thus, it's sufficient to show that

$$
n^{m} \int_{\Gamma\left(\tau^{\prime}\right)} f_{n}\left(\tau^{\prime}(\mathbf{x})\right) d \mathbf{x}=o(1) .
$$

Let $k$ be the number of edges in $\tau^{\prime}$. Then, $\tau$ has $m-k$ tree components and hence

$$
\Gamma\left(\tau^{\prime}\right)=\cup_{l=1}^{m-k} \Gamma_{l}\left(\tau^{\prime}\right) .
$$

Since $\tau^{\prime}$ is not a perfect matching, we have $m<2 k$, which implies $m-k \leq k-1$. By Lemma 3, for any $1 \leq l \leq m-k$,

$$
n^{m} \int_{\Gamma_{l}\left(\tau^{\prime}\right)} f_{n}\left(\tau^{\prime}(\mathbf{x})\right) d \mathbf{x}=o(1) \text {. }
$$

Therefore,

$$
n^{m} \int_{\Gamma\left(\tau^{\prime}\right)} f_{n}\left(\tau^{\prime}(\mathbf{x})\right) d \mathbf{x}=\sum_{l=1}^{m-k} n^{m} \int_{\Gamma_{l}\left(\tau^{\prime}\right)} f_{n}\left(\tau^{\prime}(\mathbf{x})\right) d \mathbf{x}=o(1) .
$$

For any geometric graph $H$ and any $n>0$, let $B_{n}(H)$ be the indicator for $H \subseteq G F R_{r_{n}}\left(V(H) \cup \mathcal{P}_{n}\right)$ and all edges of $H$ have length in $\left(r_{n}, R_{n}\right]$.

Lemma 6: For any $\tau \in \mathcal{T}_{m}$, if $\tau$ is a perfect matching then

$$
n^{m} \mathbf{E}\left[B_{n}\left(\tau\left(\mathcal{X}_{m}\right)\right)\right] \sim(2 \mu)^{m / 2} ;
$$

otherwise,

$$
n^{m} \mathbf{E}\left[B_{n}\left(\tau\left(\mathcal{X}_{m}\right)\right)\right]=o(1) .
$$

Proof: Consider a topology $\tau \in \mathcal{T}_{m}$. Let $k$ be the number of edges in $\tau$. Then, $k \leq m(m-1) / 2$. It's easy to verify that if $\tau$ is a perfect matching, then for any $\mathbf{x} \in \Gamma_{k}(\tau)$,

$$
\operatorname{Pr}\left[B_{n}(\tau(\mathbf{x}))=1\right]=g_{n}(\tau(\mathbf{x})) .
$$

In general, we claim that for any $\mathbf{x} \in \Gamma(\tau)$,

$$
\operatorname{Pr}\left[B_{n}(\tau(\mathbf{x}))=1\right] \leq 2^{k} f_{n}(\tau(\mathbf{x})) .
$$

Indeed, if $\chi_{r_{n}}(\tau(\mathbf{x}))=0$, then

$$
\operatorname{Pr}\left[B_{n}(\tau(\mathbf{x}))=1\right]=0
$$

and hence the claim holds trivially. Now, assume that $\chi_{r_{n}}(\tau(\mathbf{x}))=1$. Then,

$$
1 \leq \operatorname{card}(\mathcal{D}(\tau(\mathbf{x}))) \leq 2^{k} .
$$

In addition, the event $B_{n}(\tau(\mathbf{x}))=1$ implies that the geometric graph $\tau(\mathbf{x})$ has an orientation $H^{\prime} \in \mathcal{D}(\tau(\mathbf{x}))$ such that none of the regions $L_{r_{n}}(u, v)$ for all $\operatorname{arcs}(u, v)$ of $H^{\prime}$ contains any point in $\mathcal{P}_{n}$. Note that the probability of the event that none of the regions $L_{r_{n}}(u, v)$ for all arcs $(u, v)$ of an orientation $H^{\prime} \in \mathcal{D}(\tau(\mathbf{x}))$ contains any point in $\mathcal{P}_{n}$ is exactly $e^{-n\left|\left(\cup_{(u, v) \in E\left(H^{\prime}\right)} L_{r_{n}}(u, v)\right) \cap \mathbb{D}\right|}$. Therefore,

$$
\begin{aligned}
& \operatorname{Pr}\left[B_{n}(\tau(\mathbf{x}))=1\right] \\
& \leq \sum_{H^{\prime} \in \mathcal{D}(\tau(\mathbf{x}))} e^{-n\left|\left(\cup_{(u, v) \in E\left(H^{\prime}\right)} L_{r_{n}}(u, v)\right) \cap \mathbb{D}\right|} .
\end{aligned}
$$

As

$$
\nu_{r_{n}}(\tau(\mathbf{x}))=\min _{H^{\prime} \in \mathcal{D}(\tau(\mathbf{x}))}\left|\left(\cup_{(u, v) \in E\left(H^{\prime}\right)} L_{r_{n}}(u, v)\right) \cap \mathbb{D}\right|,
$$

we have

$$
\begin{aligned}
& \operatorname{Pr}\left[B_{n}(\tau(\mathbf{x}))=1\right] \\
& \leq \operatorname{card}(\mathcal{D}(\tau(\mathbf{x}))) e^{-n \nu_{r_{n}}(\tau(\mathbf{x}))} \\
& =\operatorname{card}(\mathcal{D}(\tau(\mathbf{x}))) f_{n}(\tau(\mathbf{x})) \\
& \leq 2^{k} f_{n}(\tau(\mathbf{x})) .
\end{aligned}
$$

Hence, the claim also holds if $\chi_{r_{n}}(\tau(\mathbf{x}))=1$.

Clearly,

$$
\mathbf{E}\left[B_{n}\left(\tau\left(\mathcal{X}_{m}\right)\right)\right]=\int_{\Gamma(\tau)} \operatorname{Pr}\left[B_{n}(\tau(\mathbf{x}))=1\right] d \mathbf{x} .
$$

If $\tau$ is not a perfect matching, then by Corollary 5, we have

$$
n^{m} \mathbf{E}\left[B_{n}\left(\tau\left(\mathcal{X}_{m}\right)\right)\right] \leq 2^{k} n^{m} \int_{\Gamma(\tau)} f_{n}(\tau(\mathbf{x})) d \mathbf{x}=o(1) .
$$

In the next, we assume that $\tau$ is a perfect matching. Then $m=2 k$. For $k=1, \Gamma(\tau)=\Omega$ and hence by Lemma 2 we have

$$
\begin{aligned}
& n^{2} \mathbf{E}\left[B\left(\tau\left(\mathcal{X}_{2}\right)\right)\right] \\
& =n^{2} \int_{\Omega} \operatorname{Pr}[B(\tau(\mathbf{x}))=1] d \mathbf{x} \\
& =n^{2} \int_{\Omega} g_{n}\left(x_{1}, x_{2}\right) d x_{1} d x_{2} \\
& \sim 2 \mu .
\end{aligned}
$$


So, the lemma holds for $k=1$. So, we further assume that $k \geq 2$. Note that

$$
\begin{aligned}
& n^{2 k} \mathbf{E}\left[B_{n}\left(\tau\left(\mathcal{X}_{2 k}\right)\right)\right] \\
& =n^{2 k} \int_{\Gamma(\tau)} \operatorname{Pr}\left[B_{n}(\tau(\mathbf{x}))=1\right] d \mathbf{x} \\
& =\sum_{l=1}^{k} n^{2 k} \int_{\Gamma_{l}(\tau)} \operatorname{Pr}\left[B_{n}(\tau(\mathbf{x}))=1\right] d \mathbf{x} .
\end{aligned}
$$

By Corollary 4 we have

$$
\begin{aligned}
& n^{2 k} \int_{\Gamma_{k}(\tau)} \operatorname{Pr}\left[B_{n}(\tau(\mathbf{x}))=1\right] d \mathbf{x} \\
& =n^{2 k} \int_{\Gamma_{k}(\tau)} g_{n}(\tau(\mathbf{x})) d \mathbf{x} \\
& \sim(2 \mu)^{k},
\end{aligned}
$$

and for any $1 \leq l<k$,

$$
\begin{aligned}
& n^{2 k} \int_{\Gamma_{l}(\tau)} \operatorname{Pr}\left[B_{n}(\tau(\mathbf{x}))=1\right] d \mathbf{x} \\
& \leq n^{2 k} \int_{\Gamma_{l}(\tau)} 2^{k} f_{n}(\tau(\mathbf{x})) d \mathbf{x} \\
& =o(1) .
\end{aligned}
$$

Thus,

$$
n^{2 k} \mathbf{E}\left[B_{n}\left(\tau\left(\mathcal{X}_{2 k}\right)\right)\right] \sim(2 \mu)^{k} .
$$

So, the lemma holds in this case.

For any $\mathbf{x}=\left(x_{1}, x_{2}\right) \in \mathbb{D}^{2}$, let $B_{n}^{\prime}(\mathbf{x})$ to be the indicator for $R_{n}<\left\|x_{1} x_{2}\right\| \leq R_{n}^{\prime}$ and either $L_{r_{n}}\left(x_{1}, x_{2}\right) \cap \mathcal{P}_{n}=\emptyset$ or $L_{r_{n}}\left(x_{2}, x_{1}\right) \cap \mathcal{P}_{n}=\emptyset$.

Lemma 7: $n^{2} \mathbf{E}\left[B_{n}^{\prime}\left(\mathcal{X}_{2}\right)\right]=o(1)$

Proof: For any $\mathbf{x}=\left(x_{1}, x_{2}\right) \in \Omega^{\prime}$,

$$
\operatorname{Pr}\left[B_{n}^{\prime}(\mathbf{x})=1\right]=g_{n}\left(x_{1}, x_{2}\right) .
$$

Thus,

$$
\begin{aligned}
& n^{2} \mathbf{E}\left[B_{n}^{\prime}\left(\mathcal{X}_{2}\right)\right] \\
& =n^{2} \int_{\Omega^{\prime}} \operatorname{Pr}\left[B_{n}^{\prime}(\mathbf{x})=1\right] d \mathbf{x} \\
& =\frac{n^{2}}{2} \int_{\Omega^{\prime}} g_{n}\left(x_{1}, x_{2}\right) d x_{1} d x_{2} \\
& =o(1),
\end{aligned}
$$

where the last equality follows from Lemma 2 .

\section{PROOF FOR THEOREM 1}

We first give a brief overview on our approach to prove Theorem 1. Let $M_{n}$ (respectively, $M_{n}^{\prime}$ and $M_{n}^{\prime \prime}$ ) denote the number of edges in $G F R_{r_{n}}\left(\mathcal{P}_{n}\right)$ with length in $\left(r_{n}, R_{n}\right]$ (respectively, $\left(R_{n}, R_{n}^{\prime}\right]$ and $\left.\left(R_{n}^{\prime},+\infty\right)\right)$. Then, $\phi_{n} \leq r_{n}$ if and only if $M_{n}+M_{n}^{\prime}+M_{n}^{\prime \prime}=0$. In Lemma 8, we will show that $M_{n}^{\prime \prime}=0$ is a.a.s.. In Lemma 11, we will prove that $E\left[M_{n}^{\prime}\right]=o(1)$, which implies that $M_{n}^{\prime}=0$ is a.a.s. by Markov's inequality. In Lemma 13, we will prove that $M_{n}$ is asymptotically Poisson with mean $\mu$. Consequently,

$$
\begin{aligned}
& \lim _{n \rightarrow \infty} \operatorname{Pr}\left[\phi_{n} \leq r_{n}\right] \\
& =\lim _{n \rightarrow \infty} \operatorname{Pr}\left[M_{n}+M_{n}^{\prime}+M_{n}^{\prime \prime}=0\right] \\
& =\lim _{n \rightarrow \infty} \operatorname{Pr}\left[M_{n}=0\right] \\
& =e^{-\mu} .
\end{aligned}
$$

We first utilize the tool of minimal scan statistics developed in [11] to prove that $M_{n}^{\prime \prime}=0$ is a.a.s..

Lemma 8: $\operatorname{Pr}\left[M_{n}^{\prime \prime}>0\right]=o(1)$.

Proof: For any finite point set $V \subset \mathbb{D}$ and any $r>0$, define

$$
\mathcal{S}(V, r)=\min _{(u, v) \in \mathbb{D}^{2},\|u v\|=(1+\varepsilon / 4) r}\left|V \cap L_{r}(u, v)\right| .
$$

For any $(u, v) \in \mathbb{D}^{2}$ with $\|u v\|=(1+\varepsilon / 4) r$, let $v^{\prime}$ be the point on $u v$ satisfying that $\left\|u v^{\prime}\right\|=r$. Then, there is a positive constant $\varepsilon^{\prime}>0$ such that

$$
\left|L_{r}(u, v)\right|>\left(1+\varepsilon^{\prime}\right)\left|L\left(u, v^{\prime}\right)\right| .
$$

Using this fact and following the same argument in the proof of Lemma 9 in [11], we can prove that $\mathcal{S}\left(\mathcal{P}_{n}, r_{n}\right)>\Theta(\ln n)$ is a.a.s.. In particular, $\mathcal{S}\left(\mathcal{P}_{n}, r_{n}\right)>0$ a.a.s..

Next, we claim that the event $M_{n}^{\prime \prime}>0$ implies the event $\mathcal{S}\left(\mathcal{P}_{n}, r_{n}\right)=0$. Suppose that $M_{n}^{\prime \prime}>0$. Then there are a pair of nodes $\{X, Y\} \subset \mathcal{P}_{n}$ such that $\|X Y\|>R_{n}^{\prime}$ and either $L_{r_{n}}(X, Y) \cap \mathcal{P}_{n}$ or $L_{r_{n}}(Y, X) \cap \mathcal{P}_{n}$ is empty. By symmetry, we assume that $L_{r_{n}}(X, Y) \cap \mathcal{P}_{n}$ is empty. Let $Y^{\prime}$ be the point on the ray $X Y$ satisfying that $\left\|X Y^{\prime}\right\|=(1+\varepsilon / 4) r_{n}$. When $n$ is sufficiently large, $R_{n}^{\prime}>(1+\varepsilon / 4) r_{n}$ and hence $Y^{\prime}$ is on the line segment $X Y$. Thus $L_{r_{n}}\left(X, Y^{\prime}\right) \subset L_{r_{n}}(X, Y)$. So $L_{r_{n}}\left(X, Y^{\prime}\right) \cap \mathcal{P}_{n}$ is empty. This implies that $\mathcal{S}\left(\mathcal{P}_{n}, r_{n}\right)=0$. Therefore, our claim holds.

Consequently,

$$
\operatorname{Pr}\left[M_{n}^{\prime \prime}>0\right] \leq \operatorname{Pr}\left[\mathcal{S}\left(\mathcal{P}_{n}, r_{n}\right)=0\right]=o(1) .
$$


Two key techniques used in our remaining proof are the Palm theory for Poisson processes (see, e.g., Theorem 1.6 in [8]) and the Brun's sieve (see, e.g., Theorem 10 in [12]), which are stated below.

Theorem 9: (Palm theory) Suppose that $h(U, V)$ is a bounded measurable function defined on all pairs of the form $(U, V)$ with $V$ being a finite planar set and $U$ being a subset of $V$. Then for any positive integer $k$,

$$
\mathbf{E}\left[\sum_{U \subseteq \mathcal{P}_{n},|U|=k} h\left(U, \mathcal{P}_{n}\right)\right]=\frac{n^{k}}{k !} \mathbf{E}\left[h\left(\mathcal{X}_{k}, \mathcal{X}_{k} \cup \mathcal{P}_{n}\right)\right],
$$

where $\mathcal{X}_{n}$ is the uniform $n$-point process over $\mathbb{D}$.

Theorem 10: (Brun's sieve) Let $n$ be a positive integer parameter. Suppose that $N=N(n)$ is a non-negative integer random variable depending on $n$, and $B_{1}, \cdots, B_{N}$ are $N$ Bernoulli random variables depending on $n$. If there is a constant $\mu$ such that for every fixed positive integer $k$,

$$
\mathbf{E}\left[\sum_{I \subseteq\{1, \cdots, N\},|I|=k} \prod_{i \in I} B_{i}\right] \sim \frac{\mu^{k}}{k !},
$$

then $\sum_{i=1}^{N} B_{i}$ is asymptotically Poisson with mean $\mu$ (with respect to $n \rightarrow \infty$ ).

Now, we apply Palm theory to show that $E\left[M_{n}^{\prime}\right]$ is vanishing.

Lemma 11: $E\left[M_{n}^{\prime}\right]=o(1)$.

Proof: For any pair $(U, V)$ with $V$ being a finite planar set and $U$ being a subset of $V$, define $h^{\prime}(U, V)$ to be the number of edges in $K(U)$ which have length in $\left(R_{n}, R_{n}^{\prime}\right]$ and are edges of $G F R_{r_{n}}(V)$. By applying the Palm theory (Theorem 9), we have

$$
\begin{aligned}
& \mathbf{E}\left[M_{n}^{\prime}\right] \\
& =\mathbf{E}\left[\sum_{U \subset \mathcal{P}_{n},|U|=2} h^{\prime}\left(U, \mathcal{P}_{n}\right)\right] \\
& =\frac{n^{2}}{2} \mathbf{E}\left[h^{\prime}\left(\mathcal{X}_{2}, \mathcal{X}_{2} \cup \mathcal{P}_{n}\right)\right] \\
& =\frac{n^{2}}{2} \mathbf{E}\left[B_{n}^{\prime}\left(\mathcal{X}_{2}\right)\right] .
\end{aligned}
$$

By Lemma 7, the lemma follows.

For any positive integer $k$, denote by $\mathcal{H}_{n, k}$ the collection of $k$-edge subgraphs of $G F R_{r_{n}}\left(\mathcal{P}_{n}\right)$ in which all edges have length in $\left(r_{n}, R_{n}\right]$ and no vertex is isolated. Next, we apply Palm theory to compute the asymptotic average of $\operatorname{card}\left(\mathcal{H}_{n, k}\right)$.

Lemma 12: For any fixed positive integer $k$,

$$
\mathbf{E}\left[\operatorname{card}\left(\mathcal{H}_{n, k}\right)\right] \sim \frac{\mu^{k}}{k !} .
$$

Proof: For any pair $(U, V)$ with $V$ being a finite planar set and $U$ being a subset of $V$, define $h(U, V)$ to be the number of $k$-edge subgraphs of $G F R_{r_{n}}(V)$ on $U$ in which all edges have length in $\left(r_{n}, R_{n}\right]$ and no vertex is isolated. By applying the Palm theory, we have

$$
\begin{aligned}
& \mathbf{E}\left[\operatorname{card}\left(\mathcal{H}_{n, k}\right)\right] \\
& =\mathbf{E}\left[\sum_{m=2}^{2 k} \sum_{U \subset \mathcal{P}_{n},|U|=m} h\left(U, \mathcal{P}_{n}\right)\right] \\
& =\sum_{m=2}^{2 k} \mathbf{E}\left[\sum_{U \subset \mathcal{P}_{n},|U|=m} h\left(U, \mathcal{P}_{n}\right)\right] \\
& =\sum_{m=2}^{2 k} \frac{n^{m}}{m !} \mathbf{E}\left[h\left(\mathcal{X}_{m}, \mathcal{X}_{m} \cup \mathcal{P}_{n}\right)\right] .
\end{aligned}
$$

Thus, it is sufficient to show that

$$
\frac{n^{m}}{m !} \mathbf{E}\left[h\left(\mathcal{X}_{m}, \mathcal{X}_{m} \cup \mathcal{P}_{n}\right)\right] \sim \begin{cases}0, & \text { if } 2 \leq m<2 k \\ \frac{\mu^{k}}{k !}, & \text { if } m=2 k .\end{cases}
$$

For any positive integer $k$ and any $2 \leq m \leq 2 k$, denote $\mathcal{T}_{m, k}$ the set of topologies in $\mathcal{T}_{m}$ with exactly $k$ edges. Note that any topology in $\mathcal{T}_{2 k, k}$ is a perfect matching, and

$$
\operatorname{card}\left(\mathcal{T}_{2 k, k}\right)=\frac{1}{k !}\left(\begin{array}{c}
2 k \\
2,2, \cdots, 2
\end{array}\right)=\frac{(2 k) !}{k ! 2^{k}} .
$$

By Lemma 6, we have

$$
\begin{aligned}
& \frac{n^{2 k}}{(2 k) !} \mathbf{E}\left[h\left(\mathcal{X}_{2 k}, \mathcal{X}_{2 k} \cup \mathcal{P}_{n}\right)\right] \\
& =\frac{1}{(2 k) !} \sum_{\tau \in \mathcal{T}_{2 k, k}} n^{2 k} \mathbf{E}\left[B_{n}\left(\tau\left(\mathcal{X}_{2 k}\right)\right)\right] \\
& \sim \frac{1}{(2 k) !} \cdot \frac{(2 k) !}{k ! 2^{k}} \cdot(2 \mu)^{k} \\
& =\frac{\mu^{k}}{k !} .
\end{aligned}
$$

Now suppose that $2 \leq m<2 k$. Then any topology in $\mathcal{T}_{m, k}$ is not a perfect matching. By Lemma 6 ,

$n^{m} \mathbf{E}\left[h\left(\mathcal{X}_{m}, \mathcal{X}_{m} \cup \mathcal{P}_{n}\right)\right]=\sum_{\tau \in \mathcal{T}_{m, k}} n^{m} \mathbf{E}\left[B_{n}\left(\tau\left(\mathcal{X}_{m}\right)\right)\right]=o(1)$.

Finally, we apply the Brun's sieve together with Lemma 12 to prove $M_{n}$ is asymptotically Poisson. 
Lemma 13: $M_{n}$ is asymptotically Poisson with mean $\mu$.

Proof: Let $\mathcal{E}_{n}$ be the set of edges of $K\left(\mathcal{P}_{n}\right)$. For any edge $e \in \mathcal{E}_{n}$, define $\bar{B}(e)$ to be the Bernoulli random variable which equals to one if and only if $r_{n}<\|e\| \leq R_{n}$ and $e$ is an edge of $G F R_{r_{n}}\left(\mathcal{P}_{n}\right)$. Then

$$
M_{n}=\sum_{e \in \mathcal{E}_{n}} \bar{B}(e) .
$$

For subset $F$ of $\mathcal{E}_{n}, \prod_{e \in F} \bar{B}(e)=1$ if and only if $F$ is the edge set of a subgraph of $G F R_{r_{n}}\left(\mathcal{P}_{n}\right)$ in which all edges have length in $\left(r_{n}, R_{n}\right]$ and no vertex is isolated. Fix a positive integer $k$. By treating each $k$-subset $F$ of $\mathcal{E}_{n}$ as an $k$-edge subgraph of $K\left(\mathcal{P}_{n}\right)$, we have that

$$
\sum_{F \subseteq \mathcal{E}_{n},|F|=k} \prod_{e \in F} \bar{B}(e)=\operatorname{card}\left(\mathcal{H}_{n, k}\right) .
$$

Hence, by Lemma 12,

$$
\mathbf{E}\left[\sum_{F \subseteq \mathcal{E}_{n},|F|=k} \prod_{e \in F} \bar{B}(e)\right] \sim \frac{\mu^{k}}{k !} .
$$

By Brun's sieve (Theorem 10), $M_{n}$ is asymptotically Poisson with mean $\mu$.

\section{Proof FOR LEMma 3}

In this section, we prove Lemma 3. The correctness of Lemma 3 with $l=1$ is proved in Lemma 15 , and the correctness of Lemma 3 with $l>1$ is proved in Lemma 16 . We begin with a geometric inequality used by the proof of Lemma 15.

Lemma 14: Suppose that $0<R \leq \frac{1}{200 \sqrt{\pi}}$ and $r \in$ $[R /(1+\varepsilon), R]$. Consider a geometric graph $H$ over a finite subset of $\mathbb{D}$ with at least two edges satisfying that all the edges have length between $r$ and $R$ and the midpoints of its edges induce a connected $\sqrt{3} R$-disk graph. Let $e^{\prime}$ be an edge of $H$ whose midpoint is the nearest to $\partial \mathbb{D}$, and $\ell$ be the largest distance between the midpoint of $e$ and the midpoints of other edges of $H$. Then,

$$
\sum_{e \in E(H)} \nu_{r}(e) \geq \nu_{r}\left(e^{\prime}\right)+0.0026 R \ell-16 \varepsilon R^{2} .
$$

In addition, if $\chi_{r}(H)=1$ then

$$
\nu_{r}(H) \geq \nu_{r}\left(e^{\prime}\right)+0.0026 R \ell-16 \varepsilon R^{2} .
$$

The proof of this lemma is very complicated and very lengthy. We omit the proof here due to the limitation on the space, and interested readers can find the detailed proof in [13] (see Lemma 11 in [13]).

We will frequently change the integral variables using a technique introduced in [12]. Consider a tree topology on $k$ planar points $x_{1}, x_{2}, \cdots, x_{k}$, and assume without loss of generality that $x_{k-1} x_{k}$ is an edge in this tree. Let $z_{k-1}, \rho$, and $\omega$ be the midpoint, half-length and the slope of $x_{k-1} x_{k}$ respectively. We root the tree at $x_{k}$. For $1 \leq i \leq k-2$, let $z_{i}$ be the midpoint of the edge between $x_{i}$ and its parent in such rooted tree. Then, we replace $x_{1}, x_{2}, \cdots, x_{k}$ by $z_{1}, \cdots, z_{k-1}, \rho, \omega$. The Jacobian determinant of this change is $4^{k-1} \rho$.

Lemma 15: Suppose that $2<m \leq 2 k$ and $\tau$ is a forest in $\mathcal{T}_{m}$ with $k$ edges. Then,

$$
\begin{aligned}
& n^{m} \int_{\Gamma_{1}(\tau)} f_{n}(\tau(\mathbf{x})) d \mathbf{x}=o(1), \\
& n^{m} \int_{\Gamma_{1}(\tau)} g_{n}(\tau(\mathbf{x})) d \mathbf{x}=o(1) .
\end{aligned}
$$

Proof: Enumerate the edges of $\tau$ arbitrarily by $e_{1}, \cdots, e_{k}$. For any $\mathrm{x} \in \Gamma_{1}(\tau)$, let $z_{i}$ denote the middle point of $e_{i}$ in $F(\mathbf{x})$ for each $1 \leq i \leq k$. For any pair of distinct integers $p$ and $q$ between 1 and $k$, let $S_{p q}$ denote the set of $\mathbf{x} \in \Gamma_{1}(\tau)$ satisfying that $e_{p}$ is an edge in $\tau(\mathbf{x})$ whose midpoint is the nearest to $\partial \mathbb{D}$, and $z_{q}$ is the farthest from $z_{p}$ among all $z_{1}, \cdots, z_{k}$. Then, it suffices to prove for any such $p$ and $q$,

$$
\begin{aligned}
& n^{m} \int_{S_{p q}} f_{n}(\tau(\mathbf{x})) d \mathbf{x}=o(1), \\
& n^{m} \int_{S_{p q}} g_{n}(\tau(\mathbf{x})) d \mathbf{x}=o(1) .
\end{aligned}
$$

Fix a pair of distinct integers $p$ and $q$ between 1 and $k$. Let $p^{\prime}$ and $p^{\prime \prime}$ be the indices of the two endpoints of the edges $e_{p}$. We claim that for any $\mathrm{x} \in S_{p q}$,

$$
\begin{aligned}
& f_{n}(\tau(\mathbf{x})) \leq e^{-n\left(\nu_{r_{n}}\left(x_{p^{\prime}} x_{p^{\prime \prime}}\right)+\eta_{1} R_{n}\left\|z_{p} z_{q}\right\|-\eta_{2} \varepsilon_{n} R_{n}^{2}\right),} \\
& g_{n}(\tau(\mathbf{x})) \leq 2^{k} e^{-n\left(\nu_{r_{n}}\left(x_{p^{\prime}} x_{p^{\prime \prime}}\right)+\eta_{1} R_{n}\left\|z_{p} z_{q}\right\|-\eta_{2} \varepsilon_{n} R_{n}^{2}\right),}
\end{aligned}
$$

in which $\eta_{1}=0.0026$ and $\eta_{2}=16$. Indeed, if $\chi_{r_{n}}(\tau(\mathbf{x}))=0$ then $f_{n}(\tau(\mathbf{x}))=0$ and hence the inequality (3) holds trivially. If $\chi_{r_{n}}(\tau(\mathbf{x}))=1$, then

$$
f_{n}(\tau(\mathbf{x}))=e^{-n \nu_{r_{n}}(\tau(\mathbf{x}))}
$$

and by Lemma 14,

$$
\nu_{r_{n}}(\tau(\mathbf{x})) \geq \nu_{r_{n}}\left(x_{p^{\prime}} x_{p^{\prime \prime}}\right)+\eta_{1} R_{n}\left\|z_{p} z_{q}\right\|-\eta_{2} \varepsilon_{n} R_{n}^{2},
$$


Thus, the inequality (3) also holds if $\chi_{r_{n}}(\tau(\mathbf{x}))=1$. Note that for any edge $e$ of $\tau(\mathbf{x})$,

$$
g_{n}(e) \leq 2 e^{-n \nu_{r_{n}}(e)} .
$$

Thus,

$$
\begin{aligned}
g_{n}(\tau(\mathbf{x})) & =\prod_{e \in E(\tau(\mathbf{x}))} g_{n}(e) \leq 2^{k} \prod_{e \in E(\tau(\mathbf{x}))} e^{-n \nu_{r_{n}}(e)} \\
& =2^{k} e^{-n \sum_{e \in E(\tau(\mathbf{x}))} \nu_{r_{n}}(e)}
\end{aligned}
$$

By Lemma 14, the inequality (4) holds. Therefore, we only need to show that

$$
n^{m} \int_{S_{p q}} e^{-n\left(\nu_{r_{n}}\left(x_{p^{\prime}} x_{p^{\prime \prime}}\right)+\eta_{1} R_{n}\left\|z_{p} z_{q}\right\|-\eta_{2} \varepsilon_{n} R_{n}^{2}\right)} d \mathbf{x}=o(1) .
$$

We change the integral variables $x_{1}, \cdots, x_{m}$ as follows. For the tree component containing $e_{p}$, we replace the $x_{i}$ 's in this tree by the midpoints of the edges in this tree except $z_{p}$ and $x_{p^{\prime}}, x_{p^{\prime \prime}}$ (both of which are kept). For any other tree component, we use the method introduced at the beginning of this section: pick an arbitrary edge as the rooted edge. We replace $x_{i}$ 's in this tree by the midpoints of all the edges in this tree together with the half-length and slope of the root edge. Such change of integration variables yields

$$
\begin{aligned}
& n^{m} \int_{S_{p q}} e^{-n\left(\nu_{r_{n}}\left(x_{p^{\prime}} x_{p^{\prime \prime}}\right)+c_{1} R_{n}\left\|z_{p} z_{q}\right\|-c_{2} \varepsilon_{n} R_{n}^{2}\right)} d \mathbf{x} \\
& \leq O(1) e^{c_{2} n \varepsilon_{n} R_{n}^{2}} n^{m}\left(\int_{\Omega} e^{-n \nu_{r_{n}}\left(x_{p^{\prime}} x_{p^{\prime \prime}}\right)} d x_{p^{\prime}} d x_{p^{\prime \prime}}\right) \\
& \cdot\left(\int_{\frac{r_{n}}{2}}^{\frac{R_{n}}{2}} \rho d \rho\right)^{m-k-1}\left(\int_{\mathbb{R}^{2}} e^{-c_{1} n R_{n}\left\|z_{p} z_{q}\right\|} d z_{q}\right) \\
& \cdot\left(\int_{\bar{D}\left(z_{p},\left\|z_{p} z_{q}\right\|\right)} d z\right)^{k-2} \\
& \sim O(1)(\ln n)^{\frac{o(\ln \ln n)}{\ln \ln n}} n^{m-2}\left(R_{n}^{2}-r_{n}^{2}\right)^{m-k-1} \\
& \int_{\mathbb{R}^{2}} e^{-c_{1} n R_{n}\left\|z_{p} z_{q}\right\|}\left\|z_{p} z_{q}\right\|^{2(k-2)} d z_{q} \\
& \leq O(1)(\ln n)^{\frac{o(\ln \ln n)}{\ln \ln n}} n^{m-2}\left(R_{n}^{2}-r_{n}^{2}\right)^{m-k-1} \\
& \cdot \int_{0}^{\infty} e^{-c_{1} n R_{n} \mu} \mu^{2 k-3} d \mu \\
& =O(1)(\ln n)^{\frac{o(\ln \ln n)}{\ln \ln n}} \frac{n^{m-2}\left(R_{n}^{2}-r_{n}^{2}\right)^{m-k-1}}{\left(n R_{n}\right)^{2(k-1)}} \\
& =O(1)(\ln n)^{\frac{o(\ln \ln n)}{\ln \ln n}} \frac{\left(n R_{n}^{2}-n r_{n}^{2}\right)^{m-k-1}}{\left(n R_{n}^{2}\right)^{k-1}} \\
& =O(1)(\ln n)^{\frac{o(\ln \ln n)}{\ln \ln n}} \frac{\left(c_{n}-c\right)^{m-k-1}}{(\ln n)^{k-1}}
\end{aligned}
$$

$$
\begin{aligned}
& \sim O(1)(\ln n)^{\frac{o(\ln \ln n)}{\ln \ln n}} \frac{\left(c_{n}\right)^{m-k-1}}{(\ln n)^{k-1}} \\
& =O(1) \frac{\left(c_{n}\right)^{m-k-1}}{(\ln n)^{k-1-\frac{o(\ln \ln n)}{\ln \ln n}}}=o(1),
\end{aligned}
$$

where the asymptotic equality follows from Lemma 2 , and the last equality follows from $c_{n}=o(\ln \ln n)$ and $k \geq 2$.

Lemma 16: Suppose that $2<m \leq 2 k$ and $\tau$ is a forest in $\mathcal{T}_{m}$ with $k$ edges. For any integer $2 \leq l \leq$ $\min \{m-k, k-1\}$,

$$
\begin{aligned}
& n^{m} \int_{\Gamma_{l}(\tau)} f_{n}(\tau(\mathbf{x})) d \mathbf{x}=o(1), \\
& n^{m} \int_{\Gamma_{l}(\tau)} g_{n}(\tau(\mathbf{x})) d \mathbf{x}=o(1) .
\end{aligned}
$$

Proof: $\tau$ has $m-k$ tree components on $m$ numbered vertices $v_{1}, v_{2}, \cdots, v_{m}$. Enumerate them arbitrarily by $T_{1}, \cdots, T_{m-k}$. For each $1 \leq q \leq m-k$, let

$$
I_{q}=\left\{1 \leq i \leq m: v_{i} \text { is a vertex of } T_{q}\right\}
$$

Fix an integer $2 \leq l \leq \min \{m-k, k-1\}$. Consider any nontrivial $l$-partition $\Pi=\left\{Q_{1}, Q_{2}, \cdots, Q_{l}\right\}$ of $\{1,2, \cdots, m-k\}$. It induces a partition $\Pi^{\prime}=$ $\left\{P_{1}, P_{2}, \cdots, P_{l}\right\}$ of $\{1,2, \cdots, m\}$ in which $P_{j}=\cup_{q \in Q_{j}} I_{q}$ for each $1 \leq j \leq l$. Let $S(\Pi)$ denote the set of $\mathbf{x} \in \Gamma_{l}(\tau)$ such that for each $1 \leq j \leq l$, the set of midpoints of the subgraph of $\tau(\mathbf{x})$ induced by $\left\{x_{i}: i \in P_{j}\right\}$ is a connected component of the $\sqrt{3} R_{n}$-disk graph on the midpoints of the edges in $\tau(\mathbf{x})$. Then $\Gamma_{l}(\tau)$ is the union of $S(\Pi)$ over all nontrivial $l$ partitions $\Pi$ of $\{1,2, \cdots, m-k\}$. So, it is sufficient to show that for any $l$-partition $\Pi$ of $\{1,2, \cdots, k\}$,

$$
\begin{aligned}
& n^{m} \int_{S(\Pi)} f_{n}(\tau(\mathbf{x})) d \mathbf{x}=o(1), \\
& n^{m} \int_{S(\Pi)} g_{n}(\tau(\mathbf{x})) d \mathbf{x}=o(1) .
\end{aligned}
$$

Now, fix an $l$-partition $\Pi=\left\{Q_{1}, Q_{2}, \cdots, Q_{l}\right\}$ of $\{1,2, \cdots, m-k\}$. Let $\Pi^{\prime}=\left\{P_{1}, P_{2}, \cdots, P_{l}\right\}$ be the partition of $\{1,2, \cdots, m\}$ induced by $\Pi$. For each $1 \leq j \leq l$, let $m_{j}=\operatorname{card}\left(P_{j}\right), \tau_{j}$ be the topology on $m_{j}$ numbered vertices which is a subgraph of $\tau$ induced by the subset of vertices $\left\{v_{i}: i \in P_{j}\right\}$. Then, at least one $m_{j} \geq 2$. For any $\mathbf{x}=\left(x_{1}, x_{2}, \cdots, x_{m}\right) \in \mathbb{D}^{m}$ and each $1 \leq j \leq l$, let

$$
\mathbf{x}^{(j)}=\left(x_{i_{1}}, x_{i_{2}}, \cdots, x_{i_{m_{j}}}\right)
$$


where $i_{1}, i_{2}, \cdots, i_{m_{j}}$ are the $m_{j}$ indices in $P_{j}$ in the increasing order. Clearly, for each $\mathbf{x} \in S(\Pi)$ and each $1 \leq j \leq l$, $\mathbf{x}^{(j)} \in \Gamma_{1}\left(\tau_{j}\right)$. Hence,

$$
S(\Pi) \subseteq\left\{\mathbf{x} \in \mathbb{D}^{m}: \mathbf{x}^{(j)} \in \Gamma_{1}\left(\tau_{j}\right), 1 \leq j \leq l\right\} .
$$

For any $\mathbf{x} \in S(\Pi)$,

$$
\begin{gathered}
\nu_{r_{n}}(\tau(\mathbf{x}))=\sum_{j=1}^{l} \nu_{r_{n}}\left(\tau_{j}\left(\mathbf{x}^{(j)}\right)\right), \\
\chi_{r_{n}}(\tau(\mathbf{x})) \leq \prod_{j=1}^{l} \chi_{r_{n}}\left(\tau_{j}\left(\mathbf{x}^{(j)}\right)\right),
\end{gathered}
$$

which imply

$$
f_{n}(\tau(\mathbf{x})) \leq \prod_{j=1}^{l} f_{n}\left(\tau_{j}\left(\mathbf{x}^{(j)}\right)\right) .
$$

It's obvious that for any $\mathrm{x} \in S(\Pi)$,

$$
g_{n}(\tau(\mathbf{x}))=\prod_{j=1}^{l} g_{n}\left(\tau_{j}\left(\mathbf{x}^{(j)}\right)\right) \text {. }
$$

Thus,

$$
\begin{aligned}
& n^{m} \int_{S(\Pi)} f_{n}(\tau(\mathbf{x})) d \mathbf{x} \\
& \leq n^{m} \int_{S(\Pi)} \prod_{j=1}^{l} f_{n}\left(\tau_{j}\left(\mathbf{x}^{(j)}\right)\right) d \mathbf{x} \\
& \leq n^{m} \int_{\left\{\mathbf{x} \in \mathbb{D}^{m}: \mathbf{x}^{(j)} \in \Gamma_{1}\left(\tau_{j}\right), 1 \leq j \leq l\right\}} \prod_{j=1}^{l} f_{n}\left(\tau_{j}\left(\mathbf{x}^{(j)}\right)\right) d \mathbf{x} \\
& =\prod_{j=1}^{l}\left(n^{m_{j}} \int_{\Gamma_{1}\left(\tau_{j}\right)} f_{n}\left(\tau_{j}\left(\mathbf{x}^{(j)}\right)\right) d \mathbf{x}^{(j)}\right) \\
& =o(1),
\end{aligned}
$$

where the last equality follows from Lemma 2, Lemma 15 and the fact that at least one $m_{j} \geq 2$. Similarly, we can show that

$$
n^{m} \int_{S(\Pi)} g_{n}(\tau(\mathbf{x})) d \mathbf{x}=o(1) .
$$

So, the lemma follows.
Acknowledgements: The work described in this paper was supported in part by the NSF grants CNS-0831831 and CNS0916666.

\section{REFERENCES}

[1] P. Bose, P. Morin, I. Stojmenovic, and J. Urrutia: Routing with guaranteed delivery in ad hoc wireless networks, Wireless Networks, vol. 7, pp. 609-616, 2001.

[2] G. Finn: Routing and addressing problems in large metropolitan-scale internetworks, ISI Research Report ISU/RR-87-180, March 1987.

[3] R. Jain, A. Puri, and R. Sengupta: Geographical routing using partial information for wireless ad hoc networks, IEEE Personal Communications, pp. 48-57, February 2001.

[4] B. Karp and H. Kung: GPSR: Greedy perimeter stateless routing for wireless networks, ACM MobiCom, 2000.

[5] L. Kleinrock and J. Silvester: Optimum transmission radii for packet radio networks or why six is a magic number, Proceeding of IEEE National Telecommunications Conference, 1978, pp. 431-435.

[6] E. Kranakis, H. Singh, and J. Urrutia: Compass routing on geometric networks, Proc. 11th Canadian Conference on Computational Geometry, August 1999, pp. 51-54.

[7] F. Kuhn, R. Wattenhofer, and A. Zollinger: Asymptotically optimal geometric mobile ad-hoc routing, ACM Dial-M 2002.

[8] M. Penrose: Random Geometric Graphs, Oxford University Press, 2003.

[9] H. Takagi and L. Kleinrock: Optimal transmission ranges for randomly distributed packet radio terminals, IEEE Transactions on Communications 32(3): 246-257, 1984.

[10] G. Toussaint: The relative neighborhood graph of a finite planar set, Pattern Recognition 12(4):261-268, 1980.

[11] P.-J. Wan, C.-W. Yi, F. Yao, and X. Jia: Asymptotic Critical Transmission Radius for Greedy Forward Routing in Wireless Ad Hoc Networks, $A C M$ MOBIHOC 2006, pp 25-36.

[12] P.-J. Wan, and C.-W. Yi: On The Longest Edge of Gabriel Graphs in Wireless Ad Hoc Networks, IEEE Transactions on Parallel and Distributed Systems 18(1):111-125, 2007.

[13] L. Wang, Random Geometric Aspects in Multihop Wiress Networks, PhD thesis, Department of Computer Science, Illinois Inisitite of Technology, 2010. Available online at http://www.cs.iit.edu/ $/$ wan/Thesis/LWangthesis.pdf.

[14] L. Wang, F. Yao, and C.-W. Yi: Improved Asymptotic Bounds on Critical Transmission Radius for Greedy Forward Routing in Wireless Ad Hoc Networks, ACM MOBIHOC 2008, pp. 131-138.

[15] G. Xing, C. Lu, R. Pless, and Q. Huang: On greedy geographic routing algorithms in sensing-covered networks, ACM MobiHoc 2004. 